\title{
Integrated Instrumentation and Sensor Systems Enabling Condition-Based Maintenance of Aerospace Equipment
}

\author{
Richard C. Millar \\ Department of Systems Engineering, Naval Postgraduate School, 47323 Tate Road (Bldg. 2071), \\ Patuxent River NAS, MD 20670, USA \\ Correspondence should be addressed to Richard C. Millar, rcmillar@nps.edu
}

Received 26 February 2012; Accepted 8 August 2012

Academic Editor: Nezih Mrad

Copyright ( $) 2012$ Richard C. Millar. This is an open access article distributed under the Creative Commons Attribution License, which permits unrestricted use, distribution, and reproduction in any medium, provided the original work is properly cited.

The objective of the work reported herein was to use a systems engineering approach to guide development of integrated instrumentation/sensor systems (IISS) incorporating communications, interconnections, and signal acquisition. These require enhanced suitability and effectiveness for diagnostics and health management of aerospace equipment governed by the principles of Condition-based maintenance (CBM). It is concluded that the systems engineering approach to IISS definition provided clear benefits in identifying overall system requirements and an architectural framework for categorizing and evaluating alternative architectures, relative to a bottom up focus on sensor technology blind to system level user needs. CBM IISS imperatives identified include factors such as tolerance of the bulk of aerospace equipment operational environments, low intrusiveness, rapid reconfiguration, and affordable life cycle costs. The functional features identified include interrogation of the variety of sensor types and interfaces common in aerospace equipment applications over multiplexed communication media with flexibility to allow rapid system reconfiguration to adapt to evolving sensor needs. This implies standardized interfaces at the sensor location (preferably to open standards), reduced wire/connector pin count in harnesses (or their elimination through use of wireless communications).

\section{Scope, Objectives, and Methods}

This article paper the instrumentation and sensors employed to support condition-based maintenance (CBM) of aerospace equipment, sensing the condition of equipment and components (state and operation) needed to enable diagnostics triggering necessary maintenance actions. It encompasses such instrumentation and sensors, plus the signal acquisition function and the intervening communication media, thus "integrated instrumentation/sensor systems" [IISS]. The focus is on IISS application to diagnostics and health management (DHM) enabling more cost effective CBM for the complex mechanical systems required for propulsion \& power, environmental control, flight control, and other essential functions that are weight and volume constrained, tightly integrated with other systems and vehicle structure, and exposed to the full rigors of the flight envelope and environment.
Modern aerospace equipment control systems include many control sensors that may also serve CBM functions. However, IISS are being proposed and deployed for aerospace equipment diagnostics and health management (DHM) (as described by Urban [1], Litt et al. [2], and Paris et al. [3]) to enable more effective CBM. The growing complexity of such IISS is leading to consideration of distributed DHM architectures to overcome the cost, weight, and dependability challenges of centralized system architectures.

A distinction between sensor systems, or elements of sensor systems, relates to the signal acquisition bandwidth and inherent data processing requirements. The majority of the physical parameters sensed for DHM require discrete samples at relatively low rates, on the order of 100 to 1 per second. Periodic quasistatic samples of parameters such as pressure, temperature, rotational speed, strain, position, and flow are adequate for most physical system state assessments. On the other hand, dynamic sensor measurements 
(acoustic, pressure fluctuations, vibratory motion, and strain...) require high bandwidth, high frequency records. This study addresses sensor system applications interrogating a multiplicity of diverse sensed parameters at relatively low bandwidth, but the conclusions may also apply to high bandwidth signal acquisition given appropriate multiplexing technologies.

It is hypothesized that, within the above bounds, a top down systems engineering approach will be more fruitful than a bottom up pursuit of advanced sensor technology in meeting user needs for more suitable and economic instrumentation and sensor solutions. This approach involves defining DHM IISS requirements and objectives, assessing user preferences, defining a functional architectural framework capturing the full range of alternative functional architectures, and identifying functional features and architectures that transform the performance, suitability and cost/benefit trades limiting the exploitation of advanced sensor capabilities in CBM. Relevant state of the art examples of these are briefly described.

\section{Basic Sensor System Requirements}

This section addresses the instrumentation/sensor requirements inherent to DHM IISS functionality, that is, acquisition of sensor signals indicative of equipment condition.

2.1. Accuracy, Precision, and Range. DHM may often require accurate measurements covering the full range of conditions experienced by the system in normal and abnormal operation, although in many cases thresholds identifying abnormal conditions or modes of operation may be all that is necessary. However, such thresholds may need to be field adjustable over a significant range based on service experience and high precision is required for critical measurements.

2.2. Repeatability and Stability. The operational implications of diagnostic sensor servicing or recalibration on intervals less than months and even years of service are usually unacceptable. Access to degraded sensors usually requires removal of the equipment in which it embedded. Servicing signal acquisition subsystems may not be as onerous, but it is still an undesirable cost and unavailability driver. Intermittent wire harnesses and connector faults are often the major contributor to poor repeatability of DHM sensor data. Corrupt DHM data records are common impediments to troubleshooting, prolonging, and undermining effective CBM responses.

2.3. Endurance and Reliability. Similarly, sensor and IISS dependability is crucial for diagnostic applications. Dispatch with DHM failures may be restricted, and sensors and harnesses are often inaccessible. Interconnecting harnesses are an important source of failures and false alarms that reduce mission capability and availability, and contribute to the high maintenance cost of aerospace systems.
For some specific sensor requirements in the domains considered here, for example, assessing system behavior and component state in gas turbine hot sections, sensor durability may limit the possibilities but research and development continues to expand the environmental capabilities of available sensors.

The above metrics are central to sensor selection and IISS serviceability and the overall sensor system architecture and functionality must accommodate these requirements. However, one must also consider DHM IISS requirements driven more by suitability and life cycle cost (LCC) considerations derived from the operational challenges of CBM, requirements related to the process more than the product. The sensor system must be both suitable and affordable for CBM usage. In the US Navy, CBM is expected to be implemented in accordance with the principles of reliability centered maintenance (RCM), identified as $\mathrm{CBM}+$. The most cost effective solution for individual failure modes is adopted and the cost of IISS enabled CBM must be competitive with traditional approaches.

\section{Suitability and Life Cycle Cost Objectives}

DHM IISS design considerations and requirements must consider the application and usage of the system in serving the needs of CBM.

3.1. Serviceability and Affordability. Justifying total DHM IISS life cycle cost, including maintenance, technical support and data analysis, versus the known and accepted burden of direct periodic inspection, or removal and test, of aerospace system components is a significant barrier for DHM IISS application, even when users appreciate the value of CBM. Development and deployment of DHM IISS that are serviceable and affordable in aerospace applications limits the application of CBM.

A critical factor driving cost in instrumentation and sensor systems in aerospace applications is the usual architecture of a single sensor energized by, and communicating with, unique signal acquisition circuitry over a dedicated communication channel (usually more than a few conductors per sensor). This is less common in industrial applications, where networked "smart" sensors (e.g., Madni [4]) that locally process sensor readings and communicate the results in a standard format over a digital data bus to a central data recorder are common.

The possibility of distributed sensor \& signal acquisition architectures in aerospace control system applications has been the subject of much analysis and research (e.g., see Behbahani et al. [5], Litt et al. [2], Culley and Behbahani [6], and Tulpule et al. [7]) but has been limited in application to date as the demanding aerospace operating environment and the limited thermal and vibration capabilities of available analog and digital circuit components preclude the use of such "smart" sensors.

A related issue is quality assurance for DHM IISS. Once all sensors have been installed and hooked up, verifying that the sensors are correctly hooked up and functional, 
and correcting any faults, is critical. Furthermore, DHM sensors are usually expected to be field replaceable with no change in signal acquisition calibration or compensation. Both factors drive IISS cost versus capability trades, and should be considered in selecting IISS architectures that enable embedded sensor identification and functionality checks.

3.2. Compatibility versus Intrusiveness. Sensor size, the need for access and interconnections, and signal acquisition equipment weight and volume constrain the application and utility of DHM sensor systems. Provisions for accessibility of sensors and interconnects have significant impact on equipment cost, while the need to measure more parameters, more reliably, increases DHM IISS complexity and cost. As aerospace programs proceed through design and development, with tightening cost and weight margins, the weight and cost of DHM IISS combine to drive reduction or (indefinite) deferral of CBM essential capability.

3.3. Adaptability and Flexibility. A central issue in all instrumentation/sensor systems is uncertainty, the risk that what needs to be measured to meet the system performance and reliability/availability objectives has not been anticipated or considered. Aerospace equipment is designed and developed to be robust and reliable with limited reliance on preventive and corrective maintenance. Much of the need for scheduled maintenance is in response to unanticipated equipment failure modes becoming apparent late in development and in service. Thus, the ability to reconfigure DHM IISS to acquire data not foreseen when the equipment was specified and acquired is essential for effective CBM. (Xu et al. [8] emphasize these imperatives). DHM systems should be reconfigurable in days or weeks to enable data collection for informed root cause determination and to preempt significant degradation of operability and mission availability by rapid fielding of DHM system upgrades.

3.4. User Preferences. To validate the above, a survey was sent to known DHM subject matter experts [SME], asking them to rank the above factors as contributors to DHM IISS suitability. The results of the seven responses received are displayed in Figure 1, with highest priority given to serviceability in the aerospace environment but with significant concern with user needs for easy of IISS integration with the equipment serviced and adaptability to emerging CBM requirements.

When asked to weight these system level factors, the median SME responses results were intuitively satisfactory: $1,2 / 3$, and $1 / 3$ for serviceability, unobtrusiveness, and flexibility, respectively.

\section{Functional Architecture and Implications}

4.1. DHM IISS Schematic Functional Architecture Framework. Figure 2 represents a schematic framework for DHM IISS functional architectures. It depicts a number of diverse

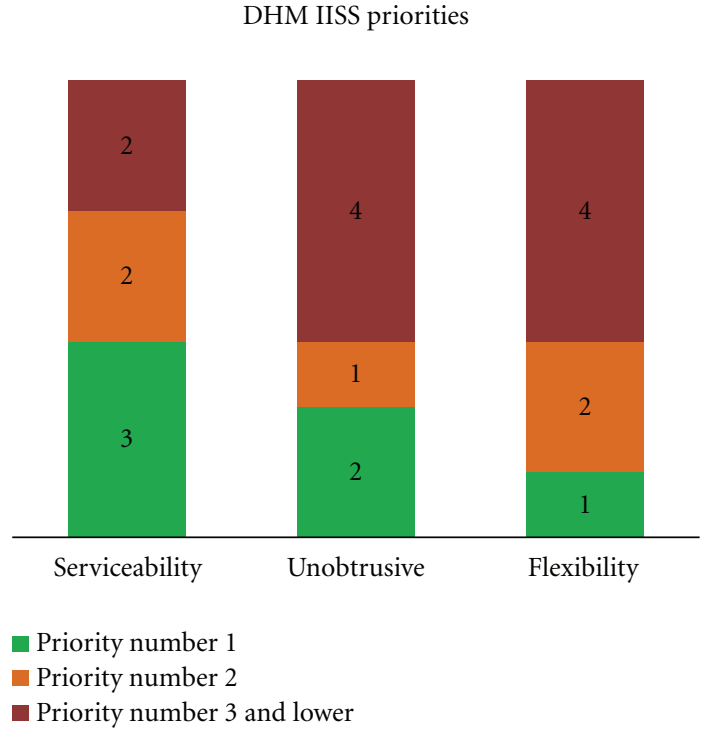

Figure 1: Subject matter expert ranking.

sensors monitoring a piece of aerospace equipment. This illustration neglects auxiliary elements, such as power supply and sensor excitation, to highlight the core functions for the purpose of identifying primary functional architectural options.

In this illustration, the state of a piece of equipment is measured by a number $(n)$ of sensors, each of which first converts a physical input into an analog input intrinsic to the physical sensor mode/design (PIN-AIn). For example, sensed pressure deflects a diaphragm to strain a strain gauge, or an optical fiber Bragg grating.

A schematic illustrating the common functions of systems for data acquisition from multiple, diverse sensors, including alternatives for multiplexed sensor data communication.

The second class of functions converts the fundamental analog input to an analog output (AIn-AOn) appropriate for signal transmission and acquisition, for example, a voltage or specific frequency of light. The third class of functions converts this analog output to a digital output (Aon-DOn), a basic function of modern a signal acquisition systems enabling further automated processing of the IISS output data (DOn) by the DHM system. These are proposed as the fundamental functional building blocks of any DHM IISS.

Furthermore, if the selection and implementation of the IISS functions are appropriate, the "digital output" originating from multiple sensors can be transmitted using a common, standardized protocol to the DHM signal acquisition and processing functions over a single multiplexed digital data bus.

If the selection and implementation of the first two functions are compatible, "analog output" originating from multiple sensors can be transmitted in a standardized format via a frequency and/or time division multiplexed channel (e.g., optical fiber or wireless analog signals sharing a common transmission medium). 


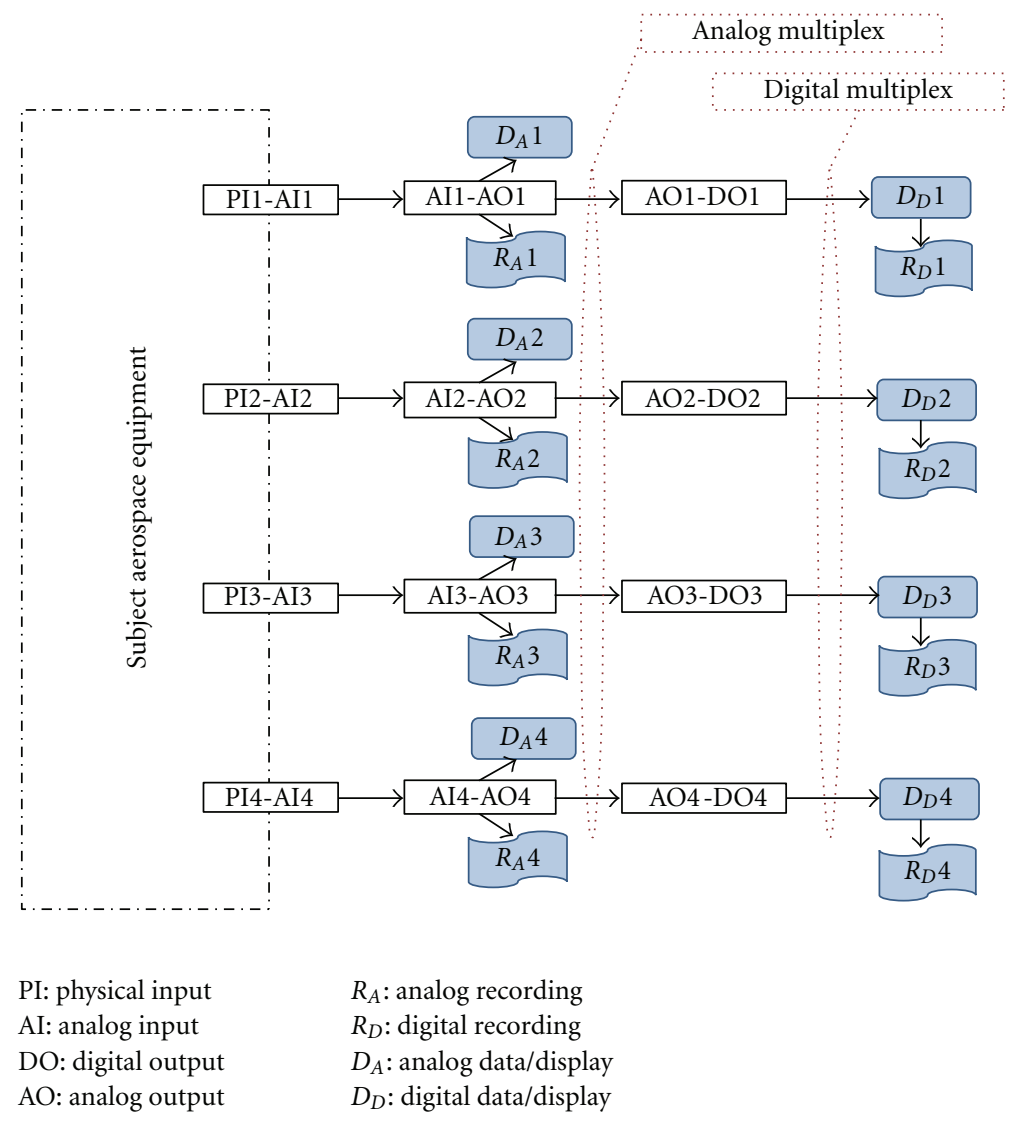

FIGURE 2: Generic functional architecture for DHM IISS.

4.2. Architectural Alternatives. The more common alternative is the standardization and multiplexed transmission of the digital outputs. This functional architecture is almost essential for state of the art DHM, where data bus based architectures for digital data transmission are the norm. However, for many aerospace applications the sensor environment and space constraints require placing the analog output signal acquisition, digital conversion, and further processing in an avionics bay or other protected environment removed from the equipment being monitored and the embedded sensors. This typically requires communication of analog sensor output over a multiplicity of dedicated channels feeding dedicated signal acquisition subsystems, curtailing the desired benefits of digital signal multiplexing: reliable, lighter, unobtrusive, and economical interconnections.

A distributed physical architecture, where the analog to digital conversion and signal multiplexing function is allocated to embedded "smart sensors", appears highly desirable. However this alternative is constrained by the environmental limitations of conventional silicon based digital electronics. Work is in progress to demonstrate a cost effective and suitable capability exploiting high temperature qualified electronic components, (see Millar and Tulpule [9] for one example).
A second architectural alternative exploits standardized analog output signals to attain the benefits of multiplexed signal transmission at this interface, as exemplified by applications of optical fiber Bragg gratings where multiple fiber optic sensors are interrogated by over a single optical fiber. (See Abad [10]). This alternative architecture requires sensors (preferable unpowered transducers) designed to conform to standardized analog outputs (electrical, optical, or radio frequency) communicated over a common transmission medium using time or wavelength division multiplexing for acquisition by a common signal acquisition channel. This is attractive in both reducing the size and complexity of harnesses and limiting the number and variety of signal acquisition interfaces and devices.

The second architecture puts severe constraints on the analog output, and demands innovation in sensor design to suit a common interface. The design disclosed in Millar [11], for example, requires that all sensors are designed such that diverse sensed physical parameters strain an optical fiber Bragg grating or otherwise modify its optical characteristics in compliance with a defined common standard, so all results can be read out with a single optoelectronic signal acquisition subsystem.

However, the latter approach is an attractive option for aerospace environments and applications where the cost, 
volume, complexity, and immaturity of robust smart sensors would otherwise put the desired benefits out of reach. (Designing sensor suites to standard analog outputs alone would enable some of the features proposed below, but the ability to multiplex the sensor analog output is necessary to radically address suitability and life cycle cost objectives).

4.3. Features Addressing Suitability and Life Cycle Cost Objectives. In general, the implication of the suitability and life cycle cost [LCC] driven objectives for integrated instrumentation/sensor system design is to make the sensors "smart" by moving the sensor specific (AOn) interface as close as possible to the sensed parameter, without compromising the sensor performance objectives. Distributed systems with standardized multiplexed interfaces at the sensor location should reduce the weight, complexity, and cost of interconnections and facilitate IISS modification in response to sensor requirements changes or capability improvements.

If the standard interface is "open," to a public specification, system capability will increase and LCC will reduce as suppliers compete to provide improved sensors meeting the standard interface, for both existing measurements and novel sensor requirements.

A capacity to support multiplexed communication will yield gains in cost and compatibility, particularly if it allows a single (smaller and less complex and costly) signal acquisition unit to interrogate multiple sensors. A variety of communication network architectures become viable, allowing optimization for enhanced system reliability and reconfiguration on the fly. As noted above, multiplexing the "analog outputs" of the sensors is the more attractive option as it may eliminate a multiplicity of differentiated signal acquisition channels. Appropriate standardization of the analog output communication protocol would enable "plug and play" sensor interchangeability, enhancing IISS adaptability to emerging DHM requirements.

Another desirable feature would be functionality for automated sensor identification (type and item) and characterization, avoiding the onerous signal tracing of conventional instrumentation, and alleviating the high costs of manufacturing sensors with effectively identical calibrations. Embedded identification and calibration (or classification) data interrogated over the signal acquisition network would be ideal, allowing plug and play sensor addition, configuration management, and interchange on the network. The nonvolatile memory function implied by this requirement might also be used to store usage, fault and system configuration data to guide maintenance.

A further desirable characteristic is a technology base shared by current and future applications in other fields, preferably ones with commercial markets that can contribute to financing technological maturation and add production volume for common components. These alternative markets would then share in recovering the sensor system investments. For example, wireless and optical fiber technology spun off from communications industry applications is a resource for development of analog multiplexed IISS.

\section{Conclusions and Recommendations}

The schematic DHM IISS functional framework presented is a useful tool to categorize alternative DHM IISS approaches. Within this framework, it will be fruitful to identify a variety of compatible implementations for each function, for example, generation of suitable pairings of analog inputs and common analog multiplex output protocols from optical fiber sensors measuring diverse physical inputs. Morphological variations on these options could be used to identify promising design alternatives for DHM IISS.

A variety of design approaches implementing the second alternative functional architecture-standardized multiplexed analog sensor outputs-have been defined and are in the early stages of feasibility demonstration and development. Six years ago, Mrad and Xiao [12] pointed out a key technology hurdle for aerospace optical fiber sensor systems: the need for robust optoelectronic signal acquisition. Luna Innovations Inc. [13] recently completed a US Navy Ph. II SBIR contract addressing this requirement.

Fonseca et al. [14] early demonstrated multiple pressure transducers responding to microwave interrogation, and Environetix [15] offered temperature sensors using a similar principle. Recent Navy SBIR topics exploring the potential of multiplexed analog signals are also bearing fruit. Gregory et al. [16] describe a SBIR project exploiting a wireless transducer concept applicable to a variety of sensor types which will allow numerous sensors in a gas turbine hot section to be wirelessly interrogated by a single signal acquisition unit. Syntonics LLC $[17,18]$ is developing a novel surface acoustic wave (SAW) wireless transducer with multiple sensor capabilities for use up to $750 \mathrm{deg}$. C.

Further effort to investigate the feasibility of, and develop, such innovative technological approaches is a promising route to more suitable and cost effective DHM IISS. In defining and evaluating such approaches, the desirable features described in the previous section should be considered. In general, the systems engineering approach provided clear benefits in defining user significant IISS system requirements and an architectural framework for categorizing, identifying, and evaluating alternative architectures, relative to a bottom up focus on sensor technology not addressing system level user needs.

\section{Nomenclature}

CBM: Condition Based maintenance

DHM: Diagnostics and health management

IISS: Integrated instrumentation/sensor systems

LCC: Life cycle cost

RCM: Reliability centered maintenance

SBIR: Small business innovation research.

\section{Acknowledgment}

The work reported here was performed for the Office of Naval Research, under the direction of Dr. Joseph Doychak. It is published here under the authority of Office of Naval Research Public Release Case no. 43-037-11. 


\section{References}

[1] L. A. Urban, "Gas path analysis applied to turbine engine conditioning monitoring," in Proceedings of the AIAA Joint Propulsion Conference, December 1972, Paper 72-1082.

[2] J. S. Litt, D. L. Simon, S. Garg et al., "A survey of intelligent control and health management technologies for aircraft propulsion systems," Journal of Aerospace Computing, Information and Communication, vol. 1, no. 12, pp. 543-563, 2004.

[3] D. E. Paris, L. C. Trevino, and M. D. Watson, "A framework for integration of IVHM technologies for intelligent integration for vehicle management," in Proceedings of the IEEE Aerospace Conference, March 2005.

[4] A. M. Madni, "Smart configurable wireless sensors and actuators for industrial monitoring and control," in Proceedings of the 3rd International Symposium on Communications, Control, and Signal Processing (ISCCSP '08), pp. 447-448, March 2008.

[5] A. Behbahani, R. C. Millar, and B. Tulpule, "Vision for next generation Modular Adaptive Generic Integrated Controls (MAGIC) for military/commercial turbine engines," in Proceedings of the AIAA Joint Propulsion Conference, pp. 69216932, July 2007, paper no 007-5710.

[6] D. E. Culley and A. Behbahani, "Communication Needs Assessment for Distributed Turbine Engine Control," NASA/TM-2008-215419, 2008.

[7] B. Tulpule, A. Behbahani, and R. Millar, Vision for Next Generation Modular Adaptive Generic Integrated Controls (MAGIC) for Military/Commercial Turbine Engines, American Institute of Aeronautics and Astronautics, 2007.

[8] X. Xu, Q. Liu, and G. Wu, "Restudying on the concept, mechanism and composition of instrumentation flexible developing system," in Proceedings of the 9th International Conference on Electronic Measurement and Instruments (ICEMI '09), pp. 3269-3274, August 2009.

[9] R. C. Millar and B. Tulpule, "Intelligent sensor node as an approach to integrated instrumentation \& sensor systems for aerospace systems control," in Proceedings of the AIAA Infotech at Aerospace, St. Louis, Mo, USA, March 2011.

[10] S. Abad, "Fiber Bragg grating sensor applications for thermal monitoring in satellites," in Proceedings of the International Conference of Space Optics, Toulouse, France, October 2008.

[11] R. C. Millar, Patent No. 7, 091, 472 B1, USA, 2006.

[12] N. Mrad and G. Z. Xiao, "Multiplexed fiber bragg gratings for potential aerospace applications," in Proceedings of the International Conference on MEMS, NANO and Smart Systems, pp. 359-363, 2005.

[13] Luna Innovations Inc., "SBIR N06-121, Open Architecture Fiber Optic Engine Monitoring System," 2010, http://www .navysbir.com/06_2/14.htm.

[14] M. A. Fonseca, J. M. English, M. von Arx, and M. G. Allen, "Wireless micromachined ceramic pressure sensor for hightemperature applications," Journal of Microelectromechanical Systems, vol. 11, no. 4, pp. 337-343, 2002.

[15] Anon, "Passive Wireless Sensor Systems for Harsh Environments, Environetics Technology Corporation," 18 July 2011, http://www.environetix.com/files/Environetix_Wireless_ Harsh_Environment_Sensors_and_Systems.pdf.

[16] O. Gregory, J. Conkle, and T. Birnbaum, "Wireless temperature sensor for gas turbine engine applications," in Proceedings of the 56th International Instrumentation Symposium, pp. 177200, Rochester, Vt, USA, May 2010.
[17] Syntonics LLC and SBIR N08-037, "High Temperature Sensing Parameters,” 2010, http://www.navysbir.com/08_1/103 .htm.

[18] J. B. Friedlander, Wireless strain measurement with surface acoustic wave sensors [M.S. thesis], Graduate School of The Ohio State University, 2011. 

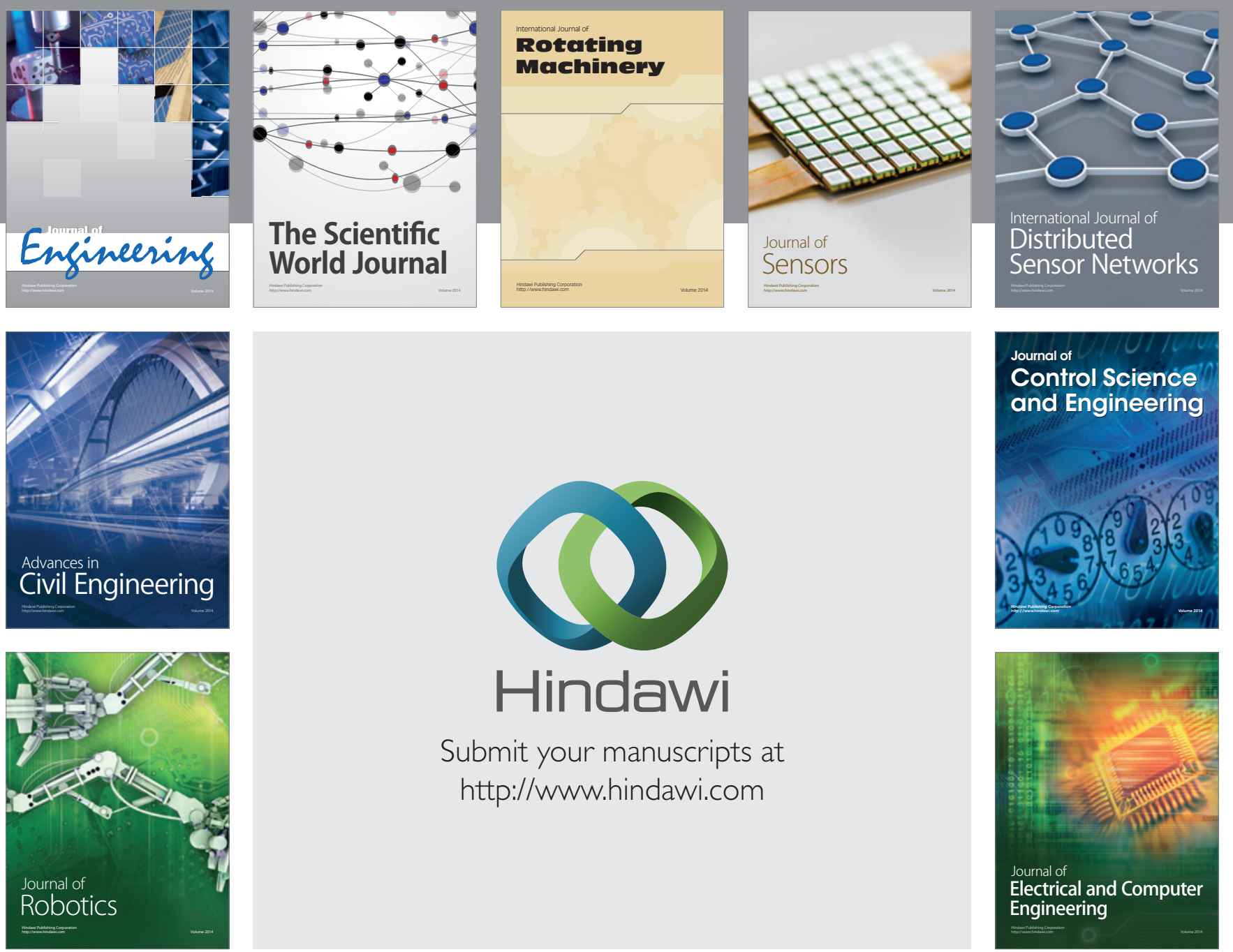

Submit your manuscripts at

http://www.hindawi.com
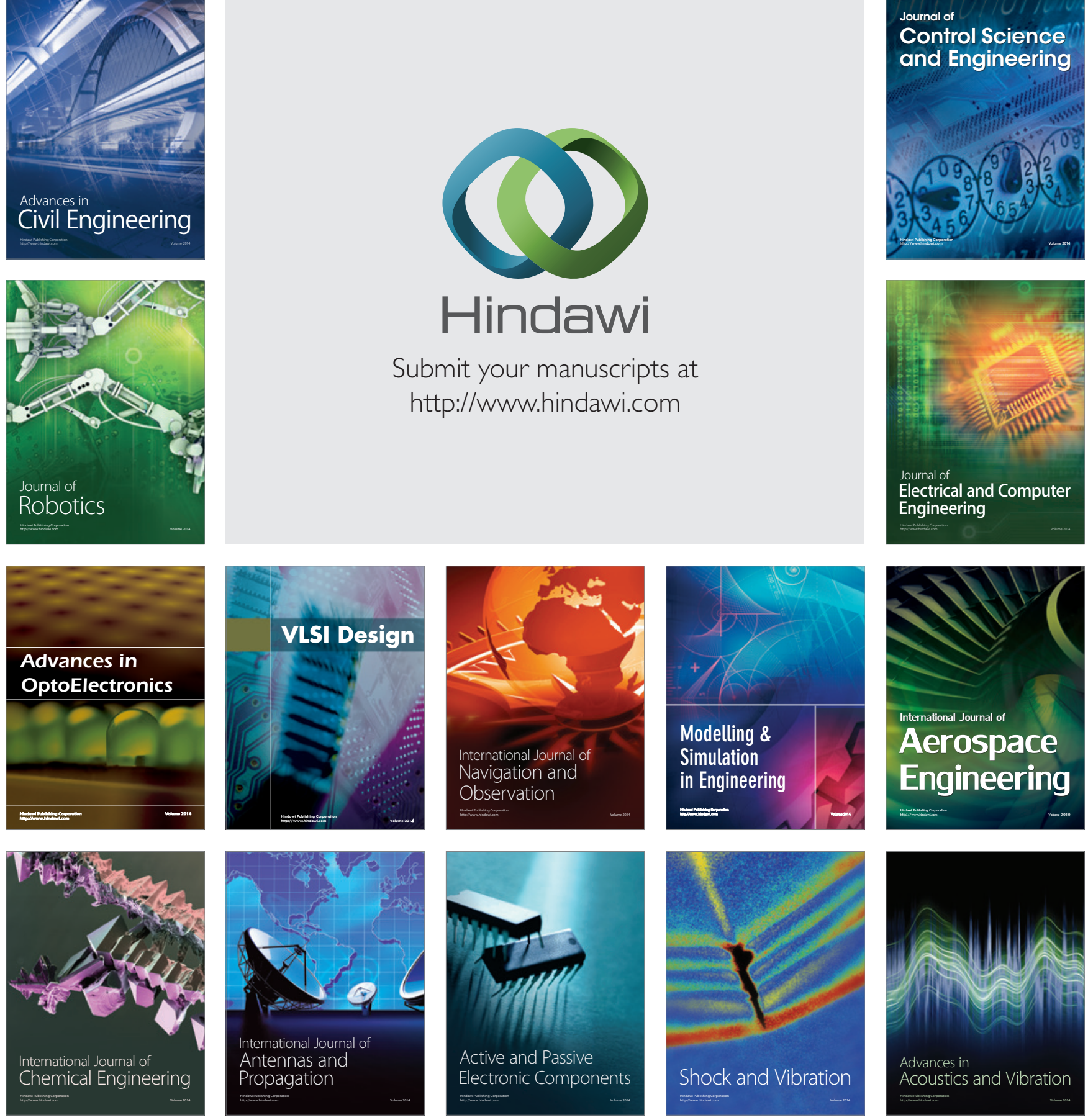